\title{
Acute infectious gastroenteritis increases likelihood of dyspepsia
}

Dyspepsia is more prevalent in people who have had an episode of acute infectious gastoenteritis than those who have not been exposed to this infection, according to results from a new Canadian study.

Acute bacterial or viral infections are known to trigger chronic lower gastrointestinal symptoms, such as IBS, in some individuals but little is known about whether these infections can also induce dyspeptic symptoms.

Alexander Ford and colleagues took advantage of a large cohort of patients from the Walkerton Health Study, which was originally instigated after an outbreak of acute infectious gastroenteritis in Walkerton, Canada, in May 2000. "Other investigators had looked at the issue of postinfective dyspepsia, but these studies were small, had limited follow-up and were inconclusive. The Walkerton cohort seemed the ideal opportunity to examine this issue further," explains Ford.
The researchers examined data from individuals at their 8-year follow-up. Of the 1,088 people assessed, $64.9 \%$ had reported acute gastroenteritis and these people were two times more likely to have dyspepsia at 8 years than people who were not exposed to gastroenteritis. Furthermore, they found that prevalence of dyspepsia was high in females, smokers, people with premorbid IBS, anxiety or depression, and in those who had diarrhea or abdominal cramps for more than a week during their acute illness.

More research is needed to determine how enteric infection affects the upper gastrointestinal tract in the long term.

Katrina Ray

Original article Ford, A. C. et al. Prevalence of uninvestigated dyspepsia 8 years after a large waterborne outbreak of bacterial dysentery: a cohort study. Gastroenterology 138, 1727-1736 (2010) 